\title{
Predictors of weaning and extubation failure in mechanically ventilated acute ischemic stroke patients
}

\author{
Yonaty $\mathrm{SA}^{1}$, Schmidt E ${ }^{1}, \mathrm{El}-$ Zammar $Z^{2}$, Elnour EM ${ }^{3}$ and Latorre $\mathrm{JG}^{1 *}$ \\ ${ }^{1}$ Neurocritical Care Service, Department of Neurology, SUNY Upstate Medical University Hospital, USA \\ ${ }^{2}$ Greater CNY Neurology Care PLLC, Syracuse, NY, USA \\ ${ }^{3}$ Neurocritical Care Service, SUNY Binghamton Campus, USA
}

\begin{abstract}
Background: Some patients with acute ischemic stroke develop respiratory failure requiring mechanical ventilation. In such patients, traditional weaning parameters poorly predict successful extubation. Failure of extubation increases medical complications. We hypothesize that predictive factors associated with weaning failure can be identified in stroke patients.
\end{abstract}

Methods: 1497 consecutive patients with acute ischemic strokes were admitted to a metropolitan academic stroke center and 158 patients (10.6\%) required mechanical ventilation. Patients who were intubated for procedures only or extubated within 48 hours (38), terminally extubated (65) or developed respiratory failure beyond acute phase (11) were excluded, leaving 44 patients for analysis.

Results: The average age was $66.4 \pm 11.8$ years, and $31(70.5 \%)$ were male. Median admission NIHSS was 14 (IQR: 10 , 19.5$)$. Seventeen patients had posterior circulation stroke (38.6\%). The overall weaning failure was 31.8\% (14/44). In multivariable analysis, acute basilar occlusion (OR=51.9 95\%CI: $2.7-992$ p<0.01) and older age (OR 1.13 95\%CI 1.01-1.27 p value 0.03) were found to be independent predictors of weaning failure. Hospital length of stay doubled (Median LOS 19 vs. 41 days), ICU length of stay tripled (Median ICU LOS 8 vs. 24 days), and total hospital cost doubled (Mean in thousands of dollars 60 vs. 122 ) in patients who failed weaning.

Conclusion: Advanced age and basilar artery occlusion were independently associated with weaning failure in acute stroke patients requiring mechanical ventilation. Weaning failure was associated with longer ICU and hospital stay doubling the cost of care. Further studies are required to verify our findings.

\begin{abstract}
Abbreviations: NIHSS: National Institutes of Health Stroke Scale; OR: Odds Ratio; CI: Confidence Interval; LOS: Length Of Stay; ICU: Intensive Care Unit; MCA: Middle Cerebral Artery; tPA: Tissue Plasminogen Activator; ASV: Adaptive Support Ventilation; SBT: Spontaneous Breathing Trial; CPAP: Continuous Positive Airway Pressure; mRS: Modified Ranking Scale; IQR: Interquartile Range; COPD: Chronic Obstructive Pulmonary Disease; UTI: Urinary Tract Infection; ANI: Acute Neurological Injury; GCS: Glasgow Coma Scale; AMS: Altered Mental Status
\end{abstract}

\section{Introduction}

Weaning mechanically ventilated patients with acute neurological injuries is challenging in the neurocritical care unit. Unlike patients in medical intensive care where oxygenation failure due to primary lung disorders are prevalent, patients with acute neurological injuries develop respiratory failure due mostly to ventilatory failure from impaired airway patency, reduced or absent protective reflexes and defective central respiratory control [1]. This difference likely explains the lack of predictive power of the traditional weaning parameters in determining the likelihood of success of extubation in the neurocritical care patients [2]. In addition, patients with neurological injury respond differently to usual weaning strategies in the intensive care, with a disproportionately high extubation failure despite passing the spontaneous breathing trial. Variable reintubation rates in neurocritically ill patients have been reported in literature and maybe related to the etiology of brain injury (as low as $5 \%$ in primarily neurosurgical and trauma patients [3], up to
$20 \%$ in patients with primarily MCA ischemic strokes [4] to as much as $36 \%$ in mixed etiology acutely brain injured patients [5]), or the location of brain injury (as high as $67 \%$ failure rate in infratentorial lesion of various etiologies [1]). Patients with primary neurological injury may have alteration in mental status leading to a low Glasgow coma scale, or inability to follow commands, which are common exclusion criteria in traditional extubation algorithms [6], complicating the decision process for ventilation weaning.

The accuracy of predicting successful extubation in mechanically ventilated patients is of paramount importance especially in critically ill patients with acute neurological injuries. In addition to increase in mortality [7] and medical complications [8], extubation failure may potentially worsen or induce hemodynamic instability, hypotension and hypoxemia leading to additional secondary brain damage ultimately resulting in poor outcome in brain injured patients. On the other hand, a number of investigators have also reported the deleterious

Correspondence to: Latorre JG, Neurocritical Care Service, Department of Neurology, SUNY Upstate Medical University Hospital, 750 East Adams Street, Syracuse, NY 13210, USA, Tel: 13154645014; Fax: 13154645015; E-mail: latorrej@upstate.edu

Key words: cerebrovascular disease, acute ischemic stroke, weaning, neurocritical care, mechanical ventilation, outcome research

Received: August 20, 2017; Accepted: September 12, 2017; Published: September 15, 2017 
effect of delaying extubation unnecessarily especially in patients with neurological injury [9].

We hypothesize that specific patient factors in acute ischemic stroke patients may be identified for predicting extubation outcome. We seek to assess the influence of stroke severity, stroke etiology, stroke location and patient admission characteristics in predicting weaning success. In addition, we determined the effect of extubation failure on long term outcome of mechanically ventilated stroke patients.

\section{Methods}

\section{Study design}

This is a retrospective study of consecutive patients admitted to a tertiary care university hospital. The study was designed and conducted following the STROBE guidelines [10]. The study proposal was reviewed and approved by the local institutional review board and informed consent was waived.

\section{Study population}

A total of 1497 patients older than 17 years and admitted with a diagnosis of acute ischemic strokes were screened. Medical records of 158 patients who were intubated within 48 hours of onset of ischemic stroke were reviewed. Patients who were intubated for procedures only or extubated within 48 hours (38), terminally extubated after withdrawal of care (65) or developed respiratory failure beyond acute phase (11) were excluded, leaving 44 patients for analysis.

\section{Patient management}

All patients included for analysis were admitted to the neurocritical care unit under a board-certified neurointensivist and managed according to published guidelines $[6,11]$. Clinical stroke severity was determined using National Institute of Health Stroke Scale (NIHSS). Patients meeting the criteria for systemic thrombolysis were given intravenous tissue plasminogen activator (IV-tPA). Patients with documented or suspected arterial thrombosis who did not improve after systemic thrombolysis (defined as post treatment $\mathrm{NIHSS}<4$ ) had emergency cerebral angiogram and mechanical thrombectomy. Decompressive craniectomy was offered to appropriate patients after discussion with health care proxy and neurosurgery attending. Mechanical ventilation was provided using adaptive support ventilation mode (ASV) and weaning was done with progressive reduction in percent minute ventilation and fraction of inspired oxygen. Once the patient met most of the traditional weaning parameters (adequate vital capacity, minute ventilation, tidal volume, respiratory rate, negative inspiratory force and frequency-tidal volume ratio) spontaneous breathing trial (SBT) was done using continuous positive airway pressure (CPAP) mode with duration and decision to extubate determined by the attending neurointensivist.

\section{Data collection}

Electronic medical records and paper charts of included patients were reviewed. Baseline clinical characteristics and demographic information were collected, including age, sex, admission NIHSS, location and etiology of cerebral infarct, location and number of occluded intracranial or extracranial vessel, past medical history of hypertension, diabetes, obesity, obstructive or restrictive pulmonary disease, ischemic cardiomyopathy, prior stroke and smoking exposure. Acute interventions including systemic and endovascularbased thrombolysis and recanalization, surgical decompression and blood transfusion were recorded. Medical complications including pneumonia (new chest x-ray findings associated with fever and positive sputum culture), seizures, sepsis, renal failure ( $>50 \%$ rise in creatinine or need for dialysis), hemorrhagic transformation, deep venous thrombosis and pulmonary embolism were identified and recorded. Duration of mechanical ventilation, fluid balance in last 24 hours, ability to follow simple commands and Glasgow coma scale immediately before extubation were collected as well.

\section{Outcome measures}

Weaning failure was defined as failure of patient to tolerate multiple attempts at spontaneous breathing trial leading to tracheostomy, or need for reintubation within 72 hours after successful extubation attempt. Dichotomized global functional outcome using modified Rankin scale (mRS) was used and determined by review of the latest outpatient records or follow-up phone call using standard modified Rankin questionnaire by a trained study member. If no records were available and contact information was missing, the modified Rankin scale at discharge determined from discharge physical therapy and neurologic examination was used. Good outcome was defined as mRS less than or equal to 3 and poor outcome was defined as mRS greater than 3. Hospital and ICU length of stay as well as total cost of hospitalization were obtained from the hospital database.

\section{Statistical analysis}

All data were analyzed with SAS for Windows software version 9.1.3 (SAS Institute Inc., Cary, NC, USA). Categorical data was analyzed using Fisher's exact test or Chi-square tests as appropriate. Continuous data with normal distribution were reported as mean \pm standard deviation and analyzed with Student's t-test, while those with non-normal distribution were reported as median (interquartile range) and analyzed with Wilcoxon 2-sample test. Multivariable adjusted logistic regression models were used to determine the independent effect of suspected predictor variables on weaning failure. For all regression models, adjustment was done by age, sex and admission stroke severity by NIHSS, in addition to variables showing a $\mathrm{p}$ value $<0.10$ in univariate analyses. Each candidate predictor variables were added in the regression model to detect changes in parameter estimate. A change of greater than $10 \%$ in the parameter estimate was considered criterion for including the variable of interest in the final model. A value of $\mathrm{p}<0.05$ was considered statistically significant.

\section{Results}

In this series, $158 / 1497(10.6 \%)$ of stroke patients required mechanical ventilation due to their acute neurological impairment. For the 44 patients included in the analysis, the mean age was 66 years (+/11.8 ), 70\% were male and median admission NIHSS score was 14 (IQR $10,19.5)$. Thirty-one $(70 \%)$ patients were intubated on admission, and the rest required intubation in the first 48 hours. Twenty-five patients had history of smoking and 9 patients had concomitant COPD. Cardioembolic stroke was the primary etiology in $50 \%$ of patients; 17 patients had posterior circulation stroke, while 13 patients had multiple vessel involvement (Table 1). Systemic thrombolysis was given in 9 patients, and 12 patients underwent emergency endovascular-based recanalization procedures. Five patients developed malignant cerebral infarction requiring surgical decompression.

\section{Successful vs. weaning failure}

Of the 44 ischemic stroke analyzed, 38 patients passed spontaneous breathing trial and 30 were successfully extubated. Eight patients failed extubation (21\%) and an additional 6 patients who failed multiple SBT 
Table 1. Patient demographics

\begin{tabular}{|c|c|c|c|c|}
\hline Patient Baseline Characteristics & $\begin{array}{c}\text { Whole Cohort } \\
\qquad \mathrm{N}=44\end{array}$ & Successful N=30 & $\begin{array}{c}\text { Failure } \\
N=14\end{array}$ & $P$ value \\
\hline Age & $66.4 \pm 11.8$ & $65.3 \pm 12.0$ & $69.1 \pm 11.3$ & $0.32^{*}$ \\
\hline Male & $31(70.4)$ & $22(73.3)$ & $9(64.3)$ & $0.54^{*}$ \\
\hline NIHSS on admission & $14(10,19.5)$ & $13.5(10,18)$ & $17.5(10,21)$ & $0.20^{*}$ \\
\hline Intubated on admission & $31970.4)$ & $20(66.7)$ & $11(78.5)$ & 0.42 \\
\hline History of prior stroke & $12(27.3)$ & $9(30)$ & $3(21.4)$ & 0.56 \\
\hline History of CAD or AF & $25(6.8)$ & $16(53.3)$ & $9(64.3)$ & 0.49 \\
\hline History of COPD & $9(20.4)$ & $4(13.3)$ & $5(35.7)$ & $0.09^{*}$ \\
\hline History of Hypertension & $37(84.0)$ & $25(83.3)$ & $12(85.7)$ & 0.84 \\
\hline History of Diabetes & $15(34.1)$ & $8(26.7)$ & $7(50)$ & $0.13^{*}$ \\
\hline Obesity & $8(18.2)$ & $4(13.3)$ & $4(28.6)$ & 0.23 \\
\hline \multicolumn{5}{|c|}{ Stroke etiology } \\
\hline Cardioembolic & $22(50)$ & $14(46.7)$ & $8(57.1)$ & 0.52 \\
\hline Large artery atherosclerosis & $21(47.7)$ & $13(43.3)$ & $8(57.1)$ & 0.39 \\
\hline \multicolumn{5}{|c|}{ Stroke location } \\
\hline Posterior circulation & $17(38.6)$ & $10(33.3)$ & $7(50)$ & 0.29 \\
\hline Basilar Artery Occlusion & $8(18.2)$ & $3(10)$ & $6(42.9)$ & $0.01^{*}$ \\
\hline Multiple vessels & $13(29.5)$ & $8(26.7)$ & $5(35.7)$ & 0.54 \\
\hline
\end{tabular}

Means \pm SD, analyzed using t-test

Median (25\%, $75 \%$ interquartile interval), analyzed using Wilcoxon 2-sample test

Number (Percent), analyzed using Fisher exact test

"Included in logistic regression model building

(NIHSS: National Institute of Health Stroke Scale; CAD : Coronary Artery Disease; AF: Atrial Fibrillation; COPD: Chronic Obstructive Pulmonary Disease)

attempts underwent tracheostomy, for a weaning failure rate of $31.8 \%$. Patients in the failure group were more likely to have history of COPD, diabetes and obesity, but the difference was not statistically significant. The median pre-extubation duration of mechanical ventilation was significantly shorter in patients who were successfully extubated (median duration 5 days (IQR 3, 7) vs. 7 (IQR 3, 17); p value 0.02 ) but did not reach statistical significance after multivariable analysis.

There was no association with extubation outcome found between stroke severity as measured by NIH Stroke Scale, stroke location (anterior vs. posterior circulation), ability to follow commands, or fluid balance. Pneumonia and UTI were more common in patients who failed weaning, while seizures and hemorrhagic transformation were more common in patients who were extubated successfully, but the difference was not statistically significant.

Using logistic regression analysis, increasing age (OR 1.13 95\%CI 1.01-1.27 p value 0.03) and presence of basilar occlusion (OR 51.9, $95 \%$ CI 2.7-992 $\mathrm{p}<0.01$ ) were found to be significant independent predictor of weaning failure, after correcting for multiple variables including stroke severity, sex and other variables in univariate screen.

Patients who failed weaning had longer ICU (median LOS in days 23.5 vs. 8 ; p value $<0.01$ ) and total hospital stay (median LOS in days 41 vs. 19 ; $\mathrm{p}$ value $<0.01)$, with higher total hospital cost $(\$ 122,000$ vs. $\$ 60,000$; $p$ value $<0.01$ ) compared to patients who were successfully extubated (Table 2).

\section{Predictors of long term outcome}

After a median follow-up of 21 months (IQR 0.5, 43), there were 11 deaths overall, including $4 / 30$ (13\%) of extubated patients, and 7/14 $(50 \%)$ of patients who failed weaning $(\mathrm{p}<0.01)$. There were 11 patients (including 5 patients with excellent outcome) in successfully extubated patients who had modified Rankin scale of $0-3$, while all survivors in the weaning failure group had moderate to severe functional disability (modified Rankin scale of 4 or 5). Successful extubation (100\% vs. $57.6 \%$; $p$ value $<0.01$ ) and shorter duration of mechanical ventilation (median in days 5 vs. 9; p value $<0.04$ ) were found to be significantly associated with good outcome in univariate screen, but did not reach significance during multivariable analysis. Interestingly, age, stroke severity and stroke location was not found to be predictor of long term outcome. Patients with poor outcome had longer hospital stay ( $28 \mathrm{vs.}$ 17 days $\mathrm{p}$ value $<0.01$ ), longer ICU stay ( 11 vs. 7 days $\mathrm{p}$ value 0.04 ) and higher hospitalization cost ( $\$ 90 \mathrm{~K}$ vs. $\$ 50 \mathrm{~K}$ p value $<0.01$ ) (Table 3 ).

\section{Early respiratory failure and patient outcome}

A significant proportion of patients required mechanical ventilation on admission (31/44 70.4\%). Patients with early respiratory failure were more likely obese with history of COPD, while patients requiring intubation in the subsequent 48 hours were more likely to have multiple vessel involvement and malignant cerebral infarction requiring surgical decompression. There was no significant difference in age, stroke severity, stroke etiology, systemic thrombolysis or endovascular thrombectomy rates among patients with early vs. late respiratory failure. After multivariable analysis, multiple vessel occlusion and the need for surgical decompression was found to be predictive of delayed respiratory failure in stroke patients.

\section{Extubation trial vs. tracheostomy}

Twelve patients underwent tracheostomy, 6 of which had initial trial of extubation. Patients who failed spontaneous breathing trial and underwent tracheostomy without trial of extubation showed trend toward shorter total mechanical ventilator days (19 vs. 26), shorter hospital days (40 vs. 43 ), shorter ICU days (21 vs. 27$)$ and less cost of hospitalization ( $\$ 116 \mathrm{~K}$ vs. $\$ 126 \mathrm{~K}$ ), although none reach statistical significance. There was no difference in long term outcome between the two groups; 6 patients ( 4 from tracheostomy group and 2 from extubation group) were dead and none of the survivors had modified Rankin scale less than 4 on follow-up. Because of limited data, no multivariable analysis was done.

\section{Discussion}

In this retrospective observational series of acute stroke patients who developed respiratory failure requiring mechanical ventilation 
Table 2. Comparison of successful vs. weaning failure patients

\begin{tabular}{|c|c|c|c|}
\hline $\begin{array}{c}\text { Clinical } \\
\text { Characteristics }\end{array}$ & Successful N=30 & Failure $N=14$ & P value \\
\hline \multicolumn{4}{|c|}{ Intervention } \\
\hline IV-tPA & $6(20)$ & $3(21.4)$ & 0.91 \\
\hline Endovascular proc & $9(30)$ & $3(21.4)$ & 0.56 \\
\hline Craniectomy & $4(14.3)$ & $1(7.1)$ & 0.55 \\
\hline Blood transfusion & $12(40)$ & $8(57.1)$ & 0.29 \\
\hline \multicolumn{4}{|c|}{ Medical Complications } \\
\hline Pneumonia & $6(20)$ & $6(42.9)$ & $0.11^{*}$ \\
\hline UTI & $8(26.7)$ & $7(50)$ & $0.13^{*}$ \\
\hline Seizure & $9(30)$ & $1(7.1)$ & $0.10^{*}$ \\
\hline Sepsis & $6(20)$ & $4(28.6)$ & 0.53 \\
\hline Renal failure & $7(23.3)$ & $5(35.7)$ & 0.39 \\
\hline $\begin{array}{l}\text { Hemorrhagic } \\
\text { transform }\end{array}$ & $14(46.6)$ & $4(28.6)$ & 0.26 \\
\hline DVT/PE & $3(10)$ & $1(7.1)$ & 0.76 \\
\hline \multicolumn{4}{|c|}{ Pre extubation parameters } \\
\hline Total MV dur (days) & $5(3,7)$ & $18.5(12,32)$ & $<0.01^{*}$ \\
\hline $\begin{array}{c}\text { Pre-extubation MV } \\
\text { days }\end{array}$ & $5(3,7)$ & $7(3,17)$ & 0.02 \\
\hline Abnormal CXR & $16(53.3)$ & $8(57.1)$ & 0.81 \\
\hline Following commands & $24(80)$ & $11(78.6)$ & 0.91 \\
\hline Need for restraint & $18(60)$ & $6(42.9)$ & 0.29 \\
\hline $\begin{array}{c}\text { Fluid input last } \\
24 \mathrm{hrs}\end{array}$ & $23(76.7)$ & $14(100)$ & $0.05^{*}$ \\
\hline \multicolumn{4}{|c|}{ Clinical Outcome } \\
\hline Hospital LOS & $19(16,25)$ & $41(38,54)$ & $<0.01$ \\
\hline ICU LOS & $8(6,10)$ & $23.5(15,33)$ & $<0.01$ \\
\hline Total Hospital Cost & $\$ 60 \mathrm{~K} \pm 24 \mathrm{~K}$ & $\$ 122 \mathrm{~K} \pm 37 \mathrm{~K}$ & $<0.01$ \\
\hline \multicolumn{4}{|l|}{$\begin{array}{l}\text { Short term } \\
\text { disposition }\end{array}$} \\
\hline Home & $3(10)$ & $2(14.3)$ & 0.68 \\
\hline Acute rehab & $17(56.7)$ & $2(14.3)$ & 0.01 \\
\hline Subacute rehab & $8(26.7)$ & $9(64.3)$ & 0.02 \\
\hline Dead & $2(6.7)$ & 0 & 0.33 \\
\hline \multicolumn{4}{|c|}{ Long term outcome } \\
\hline $\begin{array}{l}\text { Median ff-up } \\
\text { (months) }\end{array}$ & $25(0.1,47)$ & $12(2,39)$ & 0.45 \\
\hline Median mRS & $4(3,4)$ & $5.5(4,6)$ & $<0.01$ \\
\hline MRS 0 & $1(3.3)$ & 0 & \\
\hline MRS 1 & $4(13.3)$ & 0 & \\
\hline MRS 2 & 0 & 0 & \\
\hline MRS 3 & $6(20)$ & 0 & \\
\hline MRS 4 & $12(40)$ & $5(35.7)$ & \\
\hline MRS 5 & $3(10)$ & $2(14.3)$ & \\
\hline MRS 6 & $4(13.3)$ & $7(50)$ & \\
\hline
\end{tabular}

Means $\pm \mathrm{SD}$, analyzed using t-test, "included in logistic regression model building Median (25\%, 75\% interquartile interval), analyzed using Wilcoxon 2-sample test Number (Percent), analyzed using Fisher exact test

within 48 hours of symptom onset, we identified advance age and basilar artery occlusion as independent predictors of weaning failure. Older age has been associated with extubation failure in medical intensive care patients [7], while patients with infratentorial lesions due to multiple etiologies were reported to have $50 \%$ reintubation rate [1]. In our series, stroke in the posterior circulation occurred in 17 patients but was not associated with extubation outcome. However, basilar artery occlusion significantly predicted weaning failure, and maybe explained by the severity of brainstem injury affecting the respiratory centers and brainstem nuclei controlling the primary bulbar muscles responsible for maintenance of airway patency [12]. Malignant cerebral infarction requiring surgical decompression, despite substantial brain tissue loss, was not found to be significantly associated with weaning failure, nor long term outcome. In fact, four out of the five patient requiring decompression surgeries were successfully extubated and two achieved good outcome.

About $10-15 \%$ of patients in general medical intensive care who pass SBT fail, requiring reintubation [13], while up to $39 \%$ extubation failure have been reported in patients with acute neurological injuries (ANI) [14]. We found similar rate of successful SBT (38/44 86\%) and weaning failure rate $(31.8 \%)$. The disproportionately high rate of extubation failure in patients with ANI may be a result of too much reliance on traditional weaning parameters which have been shown to be poorly predictive of extubation outcome [2], or due to the unique pathophysiology of respiratory failure in ANI-inability to protect

Table 3. Predictors of long term outcome

\begin{tabular}{|c|c|c|c|}
\hline & $\begin{array}{l}\text { Good outcome } \\
\mathrm{N}=11\end{array}$ & $\begin{array}{l}\text { Poor Outcome } \\
\quad \mathrm{N}=33\end{array}$ & $P$ value \\
\hline Age & $65.3 \pm 11.9$ & $66.8 \pm 11.8$ & $0.72^{*}$ \\
\hline Male & $6(54.5)$ & $25(75.6)$ & $0.18^{*}$ \\
\hline History of Smoking & $9(81.8)$ & $16(48.4)$ & $0.08^{*}$ \\
\hline History of prior stroke & $2(18.2)$ & $10(30.3)$ & 0.43 \\
\hline History of CAD & $3(27.3)$ & $12(36.4)$ & 0.58 \\
\hline History of COPD & $2(18.2)$ & $7(21.2)$ & 0.82 \\
\hline History of Hypertension & $9(81.8)$ & $28(84.8)$ & 0.81 \\
\hline History of Diabetes & $2(18.2)$ & $13(39.4)$ & $0.19^{*}$ \\
\hline Obesity & $2(18.2)$ & $6(18.2)$ & 1.0 \\
\hline \multicolumn{4}{|c|}{ Stroke etiology } \\
\hline Cardioembolic & $4(36.4)$ & $18(54.5)$ & 0.29 \\
\hline $\begin{array}{l}\text { Large artery } \\
\text { atherosclerosis }\end{array}$ & $5(45.5)$ & $16(48.4)$ & 0.86 \\
\hline \multicolumn{4}{|c|}{ Stroke location } \\
\hline Posterior circulation & $2(18.2)$ & $15(45.4)$ & $0.10^{*}$ \\
\hline Multiple vessels & $1(9.1)$ & $12(36.4)$ & $0.13^{*}$ \\
\hline \multicolumn{4}{|c|}{ Clinical Parameters } \\
\hline NIHSS on admission & $14(10,19)$ & $14(9,20)$ & 0.95 \\
\hline Intubated on admission & $7(63.6)$ & $24(72.7)$ & 0.56 \\
\hline Admission Blood sugar & $127.6 \pm 29.3$ & $146.6 \pm 74.7$ & 0.41 \\
\hline Adm WBC & $12.2 \pm 5.2$ & $10.0 \pm 4.8$ & $0.19^{*}$ \\
\hline \multicolumn{4}{|c|}{ Intervention } \\
\hline Craniectomy & $2(18.2)$ & $3(9.1)$ & 0.41 \\
\hline IV-tPA & $3(27.3)$ & $6(18.2)$ & 0.51 \\
\hline $\begin{array}{l}\text { Endovascular } \\
\text { thrombectomy }\end{array}$ & $3(27.3)$ & $9(27.3)$ & 1.0 \\
\hline Tracheostomy & 0 & $12(36.4)$ & $0.02^{*}$ \\
\hline PEG & $1(9.1)$ & $25(75.6)$ & $<0.01^{*}$ \\
\hline Blood transfusion & $6(54.5)$ & $14(42.4)$ & 0.48 \\
\hline \multicolumn{4}{|c|}{ Clinical Outcome } \\
\hline Successful extubation & $11(100)$ & $19(57.6)$ & $<0.01^{*}$ \\
\hline Seizure & $1(9.1)$ & $9(27.3)$ & 0.21 \\
\hline Pneumonia & $1(9.1)$ & $11(33.3)$ & $0.13^{*}$ \\
\hline Sepsis & $2(18.2)$ & $8(24.2)$ & 0.67 \\
\hline Renal failure & $2(18.2)$ & $10(30.3)$ & 0.43 \\
\hline Atrial Fibrillation & $3(27.3)$ & $17(51.5)$ & $0.16^{*}$ \\
\hline $\begin{array}{l}\text { Hemorrhagic } \\
\text { transformation }\end{array}$ & $6(54.5)$ & $12(36.4)$ & 0.28 \\
\hline Intubated $>7$ days & 0 & $20(60.6)$ & $<0.01^{*}$ \\
\hline $\begin{array}{l}\text { Duration of Mech } \\
\text { ventilation }\end{array}$ & $5(3,6)$ & $9(5,17)$ & $0.04^{*}$ \\
\hline Hospital LOS & $17(12,21)$ & $28(19,40)$ & $<0.01$ \\
\hline ICU LOS & $7(6,9)$ & $11(8,20)$ & 0.04 \\
\hline Total Hospital Cost $(\$)$ & $50 \mathrm{~K} \pm 18 \mathrm{~K}$ & $90 \pm 41 \mathrm{~K}$ & $<0.01$ \\
\hline
\end{tabular}

Means +/-SD, analyzed using t-test, "included in logistic regression model building Median (25\%,75\% interquartile interval), analyzed using Wilcoxon 2-sample test Number (Percent), analyzed using Fisher exact test 
airway vs. lung pathology. In addition, clinical criteria for selecting patients appropriate for trial of extubation have usually excluded patients who have low GCS score or are unable to follow commands [6].

The impact of mental status as measured by GCS score and ability to follow commands on extubation outcome have been studied with conflicting results $[9,14,15]$. We suspect that the cause of variability in reported outcomes maybe due to the multiple etiology of altered mental status [AMS]. Patients with AMS due to supratentorial cerebral insult may have different capabilities in motor and sensory functions related to airway patency than patients with AMS due to infratentorial lesions. We tested this hypothesis by studying patients with acute stroke. Our results suggest that the degree of brainstem infarction and subsequent functional loss as determined by imaging may be important factors to consider in decision making regarding trial of extubation. Because both premature and delayed extubation carries substantial morbidities $[6,7,9]$, pre-emptive tracheostomy may reduce extubation failure and potentially reduce duration of mechanical ventilation in patients who have a low likelihood of extubation success. Among patients with infratentorial lesion, successful extubation can be expected in less than $6 \%$ of patients who were mechanically ventilated longer than 6 days $[1,16]$. Early tracheostomy in mechanically ventilated patients have been associated with reduced ICU stay [17], improved patient comfort, decreased need for sedatives [18] accelerated ventilator weaning, and reduced ventilator associated pneumonia [19], especially in trauma patients [20]. However, tracheostomy is not without complication, which includes infection, hemorrhage, displacement, obstruction, tracheal stenosis requiring repair. In addition, inappropriate tracheostomy has been associated with increased proportion of dependent survivors suffering a heavy burden of chronic disease [21]. Indeed, careful patient selection cannot be over emphasized.

Several investigators have tried to measure parameters assessing airway protection capabilities of intubated patients, including qualitative and quantitative measurement of cough strength and endotracheal secretions $[9,22,23]$ to better predict extubation outcome. A pilot study utilizing a simplified composite scoring system incorporating components of airway care for use in decision making for extubation demonstrated feasibility and safety for use in brain injured patients [24], and a large randomized study has been proposed.

The intubation rate (10.6\%) and extubation failure (21\%) in our patient cohort were similar to published literature $[4,25]$. However, we did not find any association between positive fluid balance and development of pneumonia $[15,26]$ and ability to follow commands $[15,23,27]$ with weaning outcome. In addition, stroke severity measured by NIHSS was not found to be a significant predictor of weaning outcome. This may be explained by the relatively high median NIHSS in our patient population with narrow range.

We observed $25 \%$ mortality rate in our total patient cohort, slightly better than previous reports [25]. Improvement in acute care and neurocritical care management of stroke may in part explain the observed difference. Similar to published reports, our patients who failed weaning had substantially higher mortality compared with patients successfully weaned (50\% vs. 13\%) [7]. Among survivors, 5 patients (15\%) had slight or no disability, 6 patients (18\%) had mild to moderate disability and $22(66 \%)$ had severe disability. None of the weaning failure patients had good functional outcome. It is unclear whether this may be due to factors associated with extubation failure as reported in the literature [6], or due to severity of stroke, since we are unable to verify this due to small sample size.
Consistent with other reports, we found significant increase in ICU $(3 \mathrm{x})$, hospital length of stay $(2 \mathrm{x})$, and hospital cost $(2 \mathrm{x})$ in patients who failed extubation [7].

Our study has several limitations. Because of the retrospective nature of our data collection, we are unable to verify the etiology of respiratory failure in our patient. To reduce patient heterogeneity, we assumed that patients who required mechanical ventilation on admission up to 48 hours from symptom onset developed respiratory failure as a result of the neurological compromise. Because of this we excluded patients who were intubated beyond the acute phase. Standard protocol for weaning and SBT was not available during the study period and decision to extubate, perform tracheostomy, and reintubate was made at the sole discretion of the attending neurointensivist. Moreover, there was no standard mechanical ventilation mode used and ventilator settings were determined by individual intensivists. In addition, about $20 \%$ of patients were lost to follow up and discharge outcome assessment was instead substituted for long term outcome, potentially skewing the results. We only used dichotomized global functional assessment for long term outcome. We were unable to perform quality of life measures and comprehensive neurocognitive and neuropsychiatric assessment in any of our patients. The relatively low number of patients in our series limits the validity of our results and generalizability of our findings. A prospective study with larger sample size may be necessary to verify our findings.

\section{Conclusion}

Elderly patients with respiratory failure due to acute stroke from basilar artery occlusion were more likely to fail weaning and extubation. Patients who fail extubation had longer ICU and hospital stay doubling the cost of care. Further studies are needed to determine whether preemptive tracheostomy may be beneficial in this group of patients.

\section{References}

1. Qureshi AI, Suarez JI, Parekh PD, Bhardwaj A (2000) Prediction and timing of tracheostomy in patients with infratentorial lesions requiring mechanical ventilatory support. Crit Care Med 28: 1383-1387. [Crossref]

2. Ko R, Ramos L, Chalela JA (2009) Conventional weaning parameters do not predict extubation failure in neurocritical care patients. Neurocrit Care 10: 269-273. [Crossref]

3. Navalesi P, Frigerio P, Moretti MP, Sommariva M, Vesconi S, et al. (2008) Rate of reintubation in mechanically ventilated neurosurgical and neurologic patients: evaluation of a systematic approach to weaning and extubation. Crit Care Med 36 2986-2992. [Crossref]

4. Wendell LC, Raser J, Kasner S, Park S (2011) Predictors of extubation success in patients with middle cerebral artery acute ischemic stroke. Stroke Res Treat 2011: 248789.

5. Vallverdu I, Calaf N, Subirana M, Net A, Benito S, et al. (1998) Clinical characteristics, respiratory functional parameters, and outcome of a two-hour T-piece trial in patients weaning from mechanical ventilation. Am J Respir Crit Care Med 158: 1855-1862. [Crossref]

6. MacIntyre NR, Cook DJ, Ely EW Jr, Epstein SK, Fink JB, et al. (2001) Evidence-based guidelines for weaning and discontinuing ventilatory support: a collective task force facilitated by the American College of Chest Physicians; the American Association for Respiratory Care; and the American College of Critical Care Medicine. Chest 120: 375S-395S. [Crossref]

7. Epstein SK, Ciubotaru RL, Wong JB (1997) Effect of failed extubation on the outcome of mechanical ventilation. Chest 112: 186-192.

8. Torres A, Gatell JM, Aznar E, el-Ebiary M, Puig de la Bellacasa J, et al. (1995) Reintubation increases the risk of nosocomial pneumonia in patients needing mechanical ventilation. Am J Respir Crit Care Med 152: 137-141. [Crossref]

9. Coplin WM, Pierson DJ, Cooley KD, Newell DW, Rubenfeld GD (2000) Implications of extubation delay in brain-injured patients meeting standard weaning criteria. $\mathrm{Am} \mathrm{J}$ Respir Crit Care Med 161: 1530-1536. [Crossref] 
10. von Elm E, Altman DG, Egger M, Pocock SJ, Gøtzsche PC, et al. (2007) The Strengthening the Reporting of Observational Studies in Epidemiology (STROBE) statement: guidelines for reporting observational studies. Lancet 370: 1453-1457.

11. Adams HP Jr, del Zoppo G, Alberts MJ, Bhatt DL, Brass L, et al. (2007) Guidelines for the early management of adults with ischemic stroke: a guideline from the American Heart Association/American Stroke Association Stroke Council, Clinical Cardiology Council, Cardiovascular Radiology and Intervention Council, and the Atherosclerotic Peripheral Vascular Disease and Quality of Care Outcomes in Research Interdisciplinary Working Groups: the American Academy of Neurology affirms the value of this guideline as an educational tool for neurologists. Stroke 38: 1655-1711. [Crossref]

12. Wijdicks EF, Scott JP (1996) Outcome in patients with acute basilar artery occlusion requiring mechanical ventilation. Stroke 27: 1301-1303.

13. Mokhlesi B, Tulaimat A, Gluckman TJ, Wang Y, Evans AT, et al. (2007) Predicting extubation failure after successful completion of a spontaneous breathing trial. Respir Care 52: 1710-1717

14. Namen AM, Ely EW, Tatter SB, Case LD, Lucia MA, et al. (2001) Predictors of successful extubation in neurosurgical patients. Am J Respir Crit Care Med 163: 658664. [Crossref]

15. Wang S, Zhang L, Huang K, Lin Z, Qiao W, et al. (2014) Predictors of extubation failure in neurocritical patients identified by a systematic review and meta-analysis. PLOS One 9: e112198. [Crossref]

16. Guru PK, Singh TD, Pedavally S, Rabinstein AA, Hocker S (2016) Predictors of Extubation Success in Patients with Posterior Fossa Strokes. Neurocrit Care 25: 117 127. [Crossref]

17. Koh WY, Lew TW, Chin NM, Wong MF (1997) Tracheostomy in a neuro-intensive care setting: indications and timing. Anaesth Intensive Care 25: 365-368. [Crossref]
18. Bösel J, Schiller P, Hook Y, Andes M, Neumann JO, et al. (2013) Stroke-related Early Tracheostomy versus Prolonged Orotracheal Intubation in Neurocritical Care Trial (SETPOINT): a randomized pilot trial. Stroke 44: 21-28. [Crossref]

19. Heffner JE (2001) The role of tracheotomy in weaning. Chest 120: 477S-481S [Crossref]

20. Armstrong PA, McCarthy MC, Peoples JB (1998) Reduced use of resources by early tracheostomy in ventilator-dependent patients with blunt trauma. Surgery 124: 763-766.

21. Cox CE, Carson SS, Holmes GM, Howard A, Carey TS (2004) Increase in tracheostomy for prolonged mechanical ventilation in North Carolina, 1993-2002. Crit Care Med 32: 2219-2226. [Crossref]

22. Khamiees M, Raju P, DeGirolamo A, Amoateng-Adjepong Y, Manthous CA (2001) Predictors of extubation outcome in patients who have successfully completed a spontaneous breathing trial. Chest 120: 1262-1270.

23. Salam A, Tilluckdharry L, Amoateng-Adjepong Y, Manthous CA (2004) Neurologic status, cough, secretions and extubation outcomes. Intensive Care Med 30: 1334-1339. [Crossref]

24. Manno EM, Rabinstein AA, Wijdicks EF, Brown AW, Freeman WD, et al. (2008) A prospective trial of elective extubation in brain injured patients meeting extubation criteria for ventilatory support: a feasibility study. Crit Care 12: R138.

25. Mayer SA, Copeland D, Bernardini GL, Boden-Albala B, Lennihan L, et al. (2000) Cost and outcome of mechanical ventilation for life-threatening stroke. Stroke 31: 2346-2353. [Crossref]

26. Frutos-Vivar F, Ferguson ND, Esteban A, Epstein SK, Arabi Y, et al. (2006) Risk factors for extubation failure in patients following a successful spontaneous breathing trial. Chest 130: 1664-1671. [Crossref]

27. Anderson CD, Bartscher JF, Scripko PD, Biffi A, Chase D, et al. (2011) Neurologic examination and extubation outcome in the neurocritical care unit. Neurocrit Care 15 : 490-497. [Crossref]

Copyright: (C2017 Yonaty SA. This is an open-access article distributed under the terms of the Creative Commons Attribution License, which permits unrestricted use, distribution, and reproduction in any medium, provided the original author and source are credited. 